\title{
Stable and Strong Cathodoluminescence of Fluorescent Proteins
}

Hiroki Minoda ${ }^{1}$, Tsubasa Onuma ${ }^{1}$, Naoki Yamamoto ${ }^{2}$ and Kuniaki Nagayama ${ }^{3}$

1. Tokyo University of Agriculture and Technology, 2-24-16, Naka-cho, Koganei, Tokyo, Japan

2. Tokyo Institute of Technology, 2-12-1, Ookayama, Meguro, Tokyo, Japan

${ }^{3}$ National Institute for Physiological Sciences, 5-1, Higashiyama, Myodaiji, Okazaki, Japan

Cathodoluminescence (CL) is light emission resulting from electron irradiation on luminescent materials. The CL has once been used in the cathode ray tube (CRT) screen. Inorganic fluorophores were a major player in the CRT screen owing to their robustness against the electron bombardment. Not only inorganic but also organic materials have been known to emit light by the electron irradiation. They were studied to be exploited as a high resolution biological marker with an expectation to connect the two independent regimes of light and electron microscopy. But those efforts had been hindered by low $\mathrm{CL}$ emission and rapid degradation both prone to organic substances.

A particular finding on cathodoluminescence of one type of fluorescent protein (FP) in frozen hydrated cells ${ }^{1)}$ is attractive. Actually, the first CL light from a FP species, YFP, came to us by chance beyond the original experimental plan as a byproduct in the development of a hybrid between fluorescence microscopy (FM) and electron microscopy (EM). To understand this novel finding toward a genuine CL imaging with molecular localization, we have investigated the phenomenon from following view points; i ) generality among FPs, ii ) robustness against the electron bombardment and iii) efficiency of light emission. To test generality, we worked on three kinds of FP, ${ }^{2)}$ wild type GFP, EGP and DsRed. To test robustness, the temporal change in the light emission under a continuous light and electron irradiation was monitored with the hybrid fluorescence and electron microscope mentioned $^{1)}$ and to test efficiency, SEM-CL spectroscopy was employed.

We report the microscopic and spectroscopic results with a particular focus on the stability and strength of the light emission from FPs. Possible causes of the unexpectedly stable fluorescence and cathodoluminescence emitted from FPs are to be discussed.

[1] Iijima, H., Fukuda,Y., Arai, Y., Terakawa, S., Nagayama, K., submitted to J. Struct. Biol.

[2] Tsien, R., Annu. Rev. Biochem. 37 (1998) 509-544 

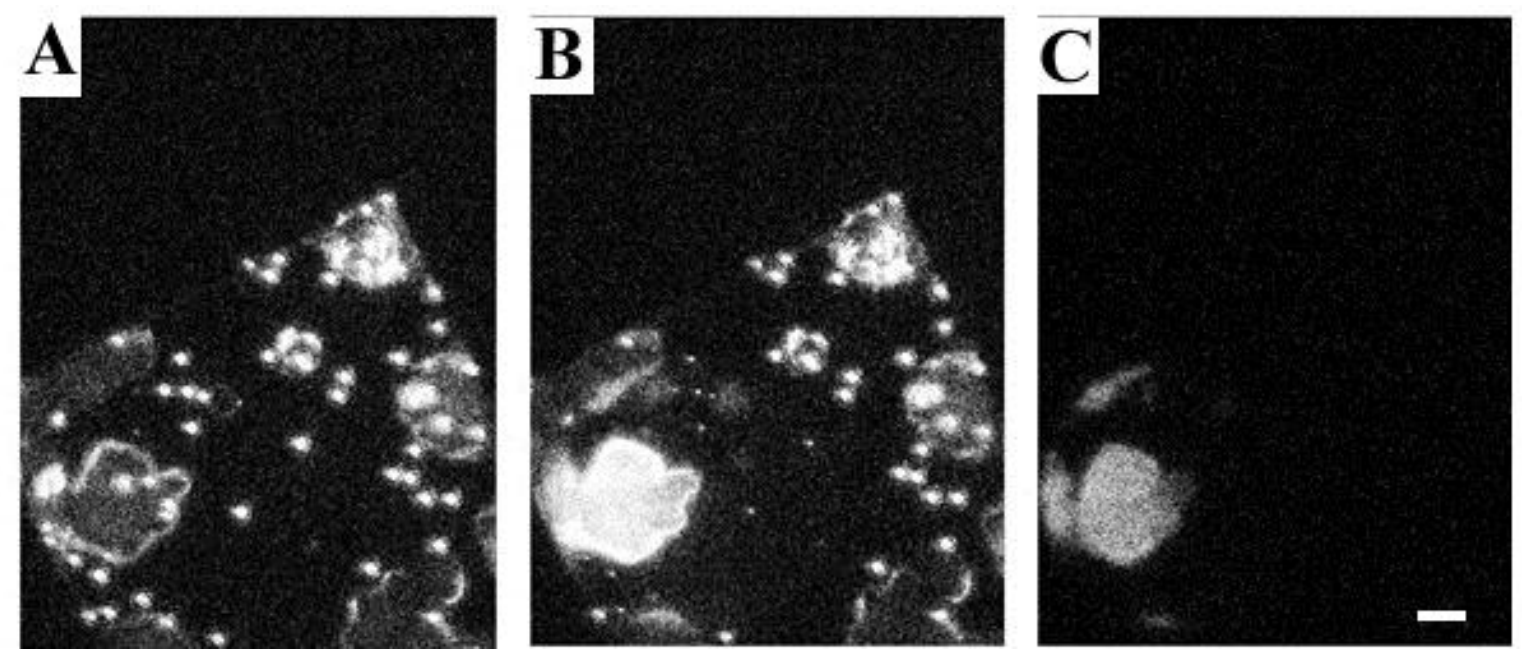

Figure 1. Three kinds of light microscopic images.

(A) An image obtained with light irradiation.

(B) An image obtained with light and electron simultaneous irradiation

(C) An image obtained with electron irradiation. Scale bar $10 \mu \mathrm{m}$.

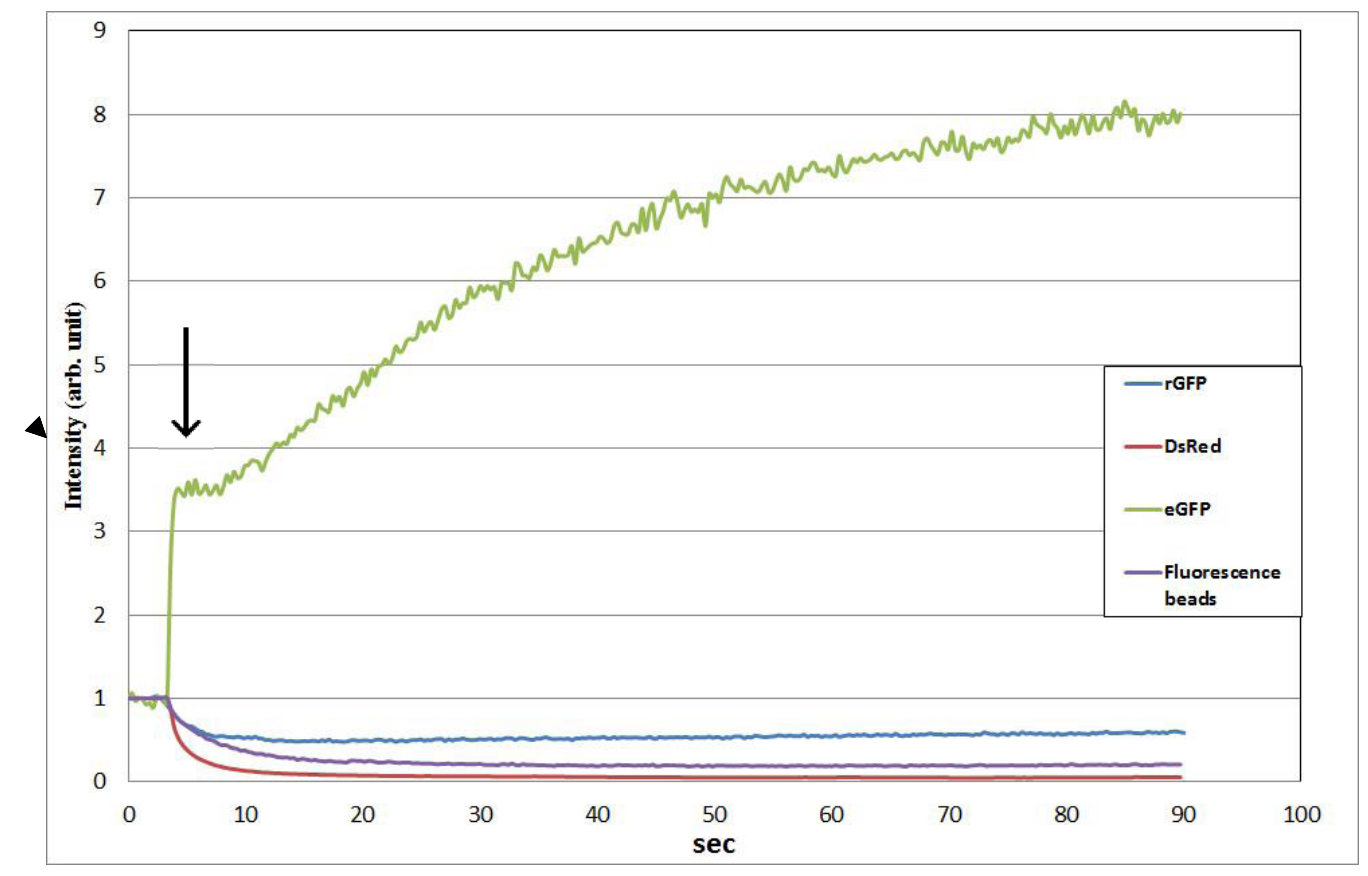

Figure 2. Temporal changes in the intensity of the light emission from various fluorescent samples under light and electron simultaneous irradiation. A sudden intensity change is brought about when an electron irradiation was switched on to illuminated samples as shown in Fig. 2 by an arrow. 